\title{
Heterogeneous Random Laser with switching activity visualized by Replica Symmetry Breaking maps
}

Loredana M. Massaro, ${ }^{\dagger}$ Silvia Gentilini, ${ }^{\ddagger}$ Alberto Portone, Aandrea Camposeo, Dario Pisignano, $§$ Claudio Conti,, and Neda Ghofraniha*,

$\dagger$ †ipartimento di Fisica, Università La Sapienza, P.le A. Moro 5, I-00185, Rome, Italy $\ddagger$ Istituto dei Sistemi Complessi-CNR, UOS Università La Sapienza, P. le A. Moro 2, I-00185, Rome, Italy

\NEST, Istituto Nanoscienze-CNR and Scuola Normale Superiore, Piazza S. Silvestro 12, I-56127 Pisa, Italy

$\S N E S T$, Istituto Nanoscienze-CNR and Dipartimento di Fisica, Università di Pisa, I-56127 Pisa, Italy

E-mail: neda.ghofraniha@cnr.it

\section{Accordance between spectral and imaging detection}

In figure S1 we report plots of the normalised emission intensity versus pumping energy from two points P1 and P2 inside the network, as shown in the related image, measured by the spectrograph and the CCD camera. Each value is an average over 100 acquisitions. The accordance is evident and the slight discrepancy is due to the different accumulation numbers used in the two techniques: images are single shot measurements and the spectra are averaged over 10 shots, being each spectrum acquired with an exposure time of 1 second. In the inset same plot in log-scale zooms on the low energy trends showing the threshold 
between spontaneous and stimulated emission. Additionally, the mean intensity trends are used in the calibration procedure to exclude sample deterioration at high energy. Indeed, in the reported example the decrease of the emission at high energy $(>7 \mu J)$ in $\mathrm{P} 1$ and $\mathrm{P} 2$ shows the irreversible dropping down of emission inside the network and is indicative of dye bleaching at high pumping. By this method we monitor point by point sample degradation in all the investigations. The analysis reported in Figure S1 is an example of calibrations we performed. Similar trends were obtained on different samples and different points, validating the experimental procedures reported in the manuscript.
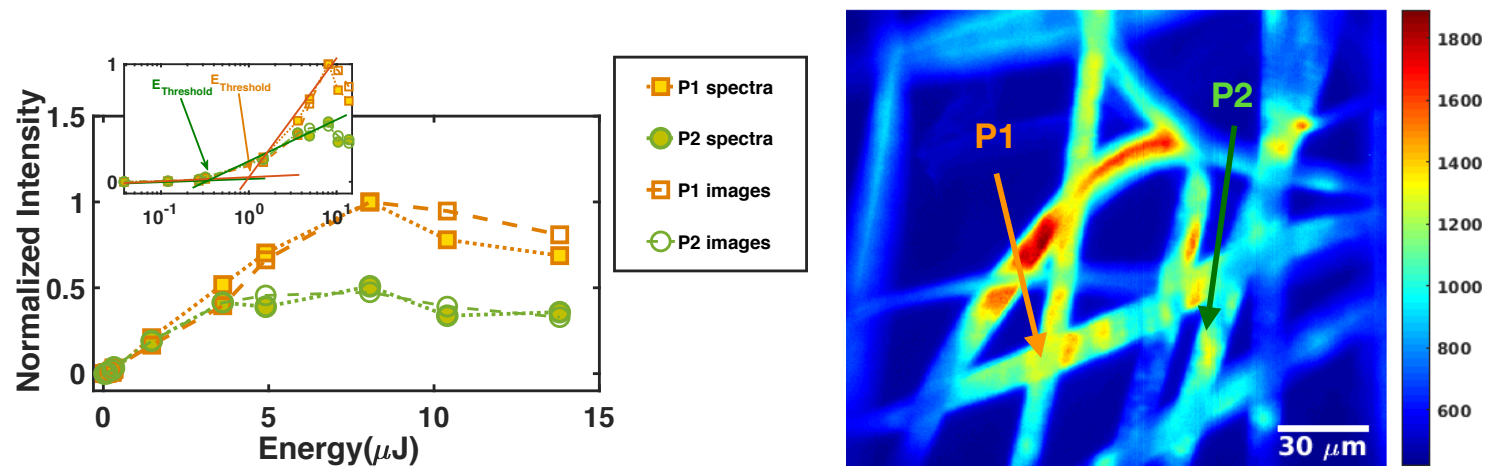

FIGURE S1: Comparison between emitted intensity taken from spectra and images on the CCD. Left: Normalised emitted intensity versus input energy from two distinct points P1 and P2 in the network, measured by the spectrograph and the CCD camera. The intensity on the spectrometer is the normalised spectral area and that from the images is the normalised intensity recorded by the pixels correspondent to P1 and P2. Inset: same plot in log-scale zooming on the low energy part and showing clearly the RL threshold of both P1 and P2. Error bars are inside the symbols. Right: The image of the selected network used for validation tests, P1 and P2 are marked. 


\section{Evidence of spatial heterogeneity in the intensity fluctuations}

The movie S2 with single shot frames shows the variability of the emission intensity fluctuations in the different points of the network at the high energy of $15.14 \mu \mathrm{J}$. Regions D1 and D2 are indicated and strong fluctuations in separated and distinct regions are signature of heterogeneous random laser. For the sake of major clarity we report three images at single shots taken from movie S2 and plots of the emission intensities in the domains D1 and D2 at different shots. It is evident that D2 has more variable pulse to pulse emissions, as also demonstrated by the calculated variances reported in figure 2 of the manuscript.
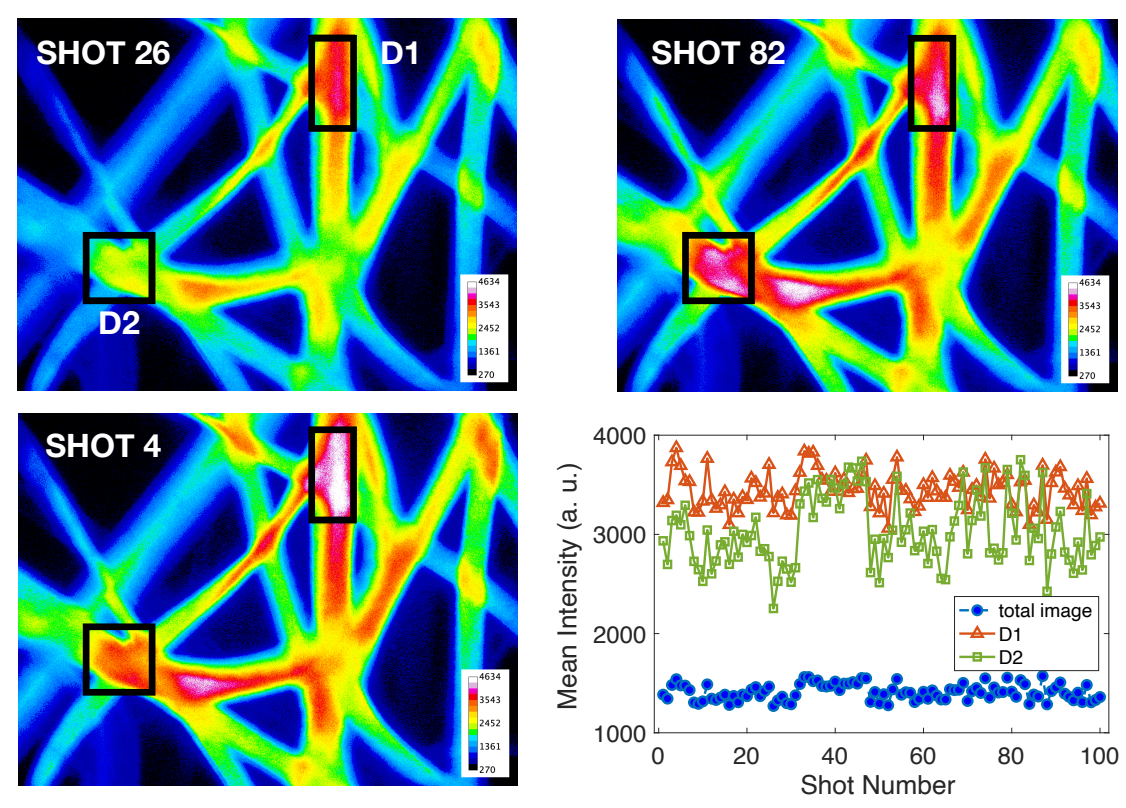

FIGURE S2: Evidence of different intensity fluctuations. Three images at single shots taken from movie S2 and plots of the emission intensity calculated from the movie S2 in regions D1, D2 and from whole the network. Error bars are inside the symbols. 